\title{
In vitro free radicals scavenging activity and antioxidant capacity of solid-state fermented wheat bran and its potential modulation of antioxidative molecular targets in chicken PBMC
}

\author{
Chaio-Chun Wang ${ }^{1}$, Chi-Huan Chang ${ }^{1}$, Shen-Chang Chang ${ }^{2}$, Geng-Jen Fan ${ }^{1,3}$, Min-Jung Lin², \\ Bi Yu', Tzu-Tai Lee ${ }^{1}$
}

${ }^{1}$ National Chung Hsing University, Department of Animal Science, Taichung, Taiwan.
${ }^{2}$ Council of Agriculture, Livestock Research Institute, Changhua Animal Propagation Station, Changhua, Taiwan.
${ }^{3}$ Council of Agriculture, Livestock Research Institute, Nutrition Division, Tainan, Taiwan.

\begin{abstract}
The purpose of this study was to demonstrate the in vitro free radical scavenging activity and antioxidant capacity in solid-state fermented wheat bran and its potential modulation of antioxidative molecular targets in chicken peripheral blood mononuclear cells (PBMC). After solid-state fermentation of wheat bran by white rot fungi for $12 \mathrm{~d}$, the scavenging action of the fermented wheat bran extracts was 1, 1-diphenyl-2-picrylhydrazyl (DPPH), and the free radicals increased significantly, approximately 1.5 -fold. Trolox equivalent antioxidant capacity of $1 \mathrm{mg} / \mathrm{mL}$ fermented wheat bran extracts was increased from 100 to $150 \mathrm{mM}$ trolox antioxidant capacity after $12 \mathrm{~d}$ of fermentation. Moreover, the extracts exhibited $50 \%$ of the chelating capacity observed for ferrous iron ( $\mathrm{Fe} 2+)$ after fermenting for $12 \mathrm{~d}$. In vitro, and under the stimulus of fermented wheat bran, the antioxidant gene expression (GST, HO-1, Nrf2, and GCLC genes) of PBMC was more than double that of the PBS, ascorbic acid, and unfermented wheat bran. The expression of fermented wheat bran was the lowest for the NOX1 and ROMO1 genes. Solid-state wheat bran fermented by white rot fungi can increase the scavenging action of DPPH, the trolox equivalent antioxidant capacity, and the chelating capacity of ferrous iron; in addition, in vitro, it can regulate the expression of antioxidant molecular targets in chicken PBMC.
\end{abstract}

Key Words: broiler, feed additive, white rot fungi

\section{Introduction}

In recent years, the rapidly growing global population has considerably increased the demand for grain. As a result, the cost of raw materials has increased significantly for dietary grain. Finding alternative feeds has become an important task for the feed industry. Processing raw plant materials may generate large amounts of by-products, such as oil cakes, along with higher seed costs. The nutritional utilization of feed is limited in the diet of monogastric animals because it is difficult for these animals to degrade its high content of lignocellulosic biomass (Yu et al., 2008). The annual global production of wheat is over 6.5 million tons, and bran is the main by-product of wheat flour processing. It is easy to reduce feed intake and growth performance because of the low energy of wheat bran (approximately $1,300 \mathrm{kcal} / \mathrm{kg}$ of metabolizable energy) and its percentage of dietary fiber (44.0\%) (Dale, 1996; Prückler et al., 2014).

Received January 13, 2016 and accepted May 3, 2016.

Corresponding author: ttlee@dragon.nchu.edu.tw

http://dx.doi.org/10.1590/S1806-92902016000800005

Copyright (c) 2016 Sociedade Brasileira de Zootecnia. This is an Open Access article distributed under the terms of the Creative Commons Attribution License (http://creativecommons.org/licenses/by/4.0/), which permits unrestricted use, distribution, and reproduction in any medium, provided the original work is properly cited.
White rot fungi is a group of filamentous fungi which could degrade lignocellulosic biomass. Its fruiting body and stalk residue (likely Pleurotus eryngii) contain high antioxidant and anti-inflammatory properties that can enhance the oxidative status of poultry (Lee et al., 2012; Lee et al., 2014; Lin et al., 2014b). In addition to the secretion of lignocellulosic enzyme, white rot fungi contain rich secondary metabolites. These ingredients could protect cells from the damage of free radicals (Sahin et al., 2008; Tuzcu et al., 2008; Sahin et al., 2010) because these metabolites can activate antioxidant-related transcription factors such as the Nuclear Factor-Erythroid 2-Related Factor 2 (Nrf2)/ Kelch-like ECH-associated protein 1 (Keap1) and induce self-defense and the expression of antioxidant genes (Sahin et al., 2013). Although the applications of wheat bran have significantly increased in recent years, its main use is to replace corn in dietary feedstuff (Dale, 1996; Prückler et al., 2014). Recent studies have attempted to use fungal inoculum to improve the nutritional value of feed (Yang et al., 2013). To our knowledge, little information is available regarding the in vitro free radical scavenging activity of solid-state fermented wheat bran and its potential modulation of antioxidative molecular targets in chicken peripheral blood mononuclear cells (PBMC). 
Therefore, in the current study, we selected white rot fungi from Pleurotus eryngii for the fermentation of wheat bran to evaluate the in vitro oxidative status modulation of antioxidant molecular targets against chicken PBMC.

\section{Material and Methods}

Pleurotus eryngii spent mushroom compost was kindly provided by a local mushroom producer (Q-YO Bio-technology Farm, Changhua, Taiwan). The P. eryngii spent mushroom compost (approximately $1 \mathrm{~cm}^{3}$ ) was grown in a bed-packed incubator at $28{ }^{\circ} \mathrm{C}$ for $3 \mathrm{~d}$ before wheat bran inoculation. Following this period, the wheat bran was used as a substrate and inoculated with $P$. eryngii particles at a ratio of 9:1 to conduct solid-state fermentation for $12 \mathrm{~d}$ (with $60 \%$ moisture at $30^{\circ} \mathrm{C}$ ). After fermentation, the solid product was dried at $70{ }^{\circ} \mathrm{C}$ for $24 \mathrm{~h}$ to reduce the moisture content to approximately $10 \%$. The extracts of fermented feed were added to $100 \%$ distilled water $(1: 10$, $\mathrm{w} / \mathrm{v}$ ) at $95^{\circ} \mathrm{C}$ for $2 \mathrm{~h}$ after filtering (Advantec No. 1, Tokyo, Japan). The filtrate was evaporated until dry under vacuum conditions, and the lyophilized extracts were added to the aqueous solution and adjusted to $1 \mathrm{mg} / \mathrm{mL}$ for advanced analysis.

The superoxide anion scavenging activity of fermented wheat bran extracts was based on the method described by Nishikimi (1972). The superoxide radicals were generated in a Tris- $\mathrm{HCl}$ buffer $(16 \mathrm{mM}, \mathrm{pH} 8.0)$ containing $600 \mu \mathrm{M}$ of nitroblue tetrazolium (NTB) and $1872 \mu \mathrm{M}$ of dihydrom icotineamidadenibe dinucleotide (NADH), and the solution of fermented wheat bran extracts was then added. For the reaction, $240 \mu \mathrm{M}$ of Phenazine methosulfate (PMS) were added to the mixture, which was left standing at room temperature for $6 \mathrm{~min}$. The absorbance was measured at $560 \mathrm{~nm}$ in a spectrophotometer, with PBS used as control. Decreased absorbance of the reaction mixture indicated increased superoxide anion scavenging capacity. The inhibition percentage of superoxide anion generation was calculated as follows:

Inhibition superoxide anion generation $(\%)=100-$

$$
[(\mathrm{A} 0-\mathrm{A} 1 / \mathrm{A} 0) \times 100] \text {, }
$$

in which $\mathrm{A} 0$ is the absorbance of the control (PBS); and A1 is the absorbance of the fermented wheat bran extracts.

The free radical scavenging activity of fermented wheat bran extracts was measured via DPPH, according to the method outlined by Blois (1958). Briefly, $0.1 \mathrm{mM}$ solution of DPPH in ethanol was prepared, and fermented wheat bran extracts were added at different concentrations. After $20 \mathrm{~min}$, absorbance was measured at $517 \mathrm{~nm}$, and PBS was used as control. Lower absorbance of the reaction mixture indicated a higher free radical scavenging effect and was calculated by:

DPPH scavenging effects $(\%)=100-[(\mathrm{A} 0-\mathrm{A} 1 / \mathrm{A} 0) \times 100]$, in which $\mathrm{A} 0$ is the absorbance of the control reaction (PBS), and $\mathrm{A} 1$ is the absorbance in the presence of fermented wheat bran extracts.

Trolox equivalent antioxidant capacity was measured according to the method described by Gyamfi et al. (1999), by mixing $0.25 \mathrm{~mL}$ of peroxidase $(4.4 \mathrm{U} / \mathrm{mL}), 0.25 \mathrm{~mL}$ of $\mathrm{H}_{2} \mathrm{O}_{2}(50 \mu \mathrm{m})$, and $1.5 \mathrm{~mL}$ of distilled water into the TEAC reagent. Spectrophotometry was used to measure the absorbance change after the reaction at $750 \mathrm{~nm}$. Results were calculated and plotted with respect to concentrations of ascorbic acid (vitamin C) and fermented wheat bran extracts, expressed as $\mathrm{mM}$ of Trolox antioxidant equivalent per gram.

The ferrous ions chelation activity of fermented wheat bran extracts and EDTA was investigated according to the method of Dinis et al. (1994). Briefly, fermented wheat bran extracts were added to a solution of $2.0 \mathrm{mM}$ ferrous chloride solution and methanol. Then, the reaction was initiated by the addition of $5.0 \mathrm{mM}$ ferrozine and left at room temperature $\left(25^{\circ} \mathrm{C}\right)$ for $12 \mathrm{~min}$. When the reaction was completed, the absorbance of the mixture was measured spectrophotometrically at $562 \mathrm{~nm}$. The inhibiting percentage of ferrozine- $\mathrm{Fe}^{2+}$ complex formation was calculated according to the following formula:

Ferrous ion chelating $(\%)=[(\mathrm{A} 0-\mathrm{A} 1) / \mathrm{A} 0] \times 100$, in which $\mathrm{A} 0$ was the absorbance of the control; and $\mathrm{A} 1$ was the absorbance of the fermented wheat bran and EDTA. The control contained ferrous chloride, ferrozine, and complex formation molecules.

Peripheral blood mononuclear cells (PBMC) were isolated from broilers (35 days old), and plasma was added at $1 \%$ ethylenediaminetetraacetic acid (EDTA) for anticoagulant treatment before being processed via density gradient centrifugation. Peripheral blood mononuclear cells were separated out in Histopaque ${ }^{\circledR}-1077$ (Sigma, 10771) and cultured in RPMI-1640 with addition of $100 \mu \mathrm{g} / \mathrm{mL}$ penicillin, $100 \mu \mathrm{g} / \mathrm{mL}$ streptomycin, and $50 \mu \mathrm{g} / \mathrm{mL}$ amphotericin at $37{ }^{\circ} \mathrm{C}$ in $5 \% \mathrm{CO}_{2}$ mixed with $95 \%$ air in an incubator as described by Kaiser et al. (2006).

For the determination of mRNA expression levels from the chicken PBMC treated with fermented wheat bran, the total RNA was isolated from plasma using the SuperScriptTM First-Strand Synthesis System reagent (Invitrogen, USA) according to the protocol given by the manufacturer. Total RNA was isolated from cultured PBMC using the Trizol reagent (Invitrogen, USA) according to the protocol of the manufacturer for the determination of 
mRNA expression levels. Determinations of total RNA concentration and purity, cDNA synthesis, and qPCR analysis were performed as described by Lin et al. (2014a). The design of Gene-specific the primers was according to genes of Gallus gallus (from chicken) (Table 1). After normalization of gene expression data using the calculated GeNorm normalization factor, the means and standard deviation were calculated from normalized expression data for samples of the same treatment.

The data were analyzed by performing ANOVA for completely randomized designs using the GLM procedure of the SAS (Statistical Analysis System, version 9.4) software. Significant differences among the various treatment group means were determined using Duncan's multiple range test. A threshold value of $\mathrm{P}<0.05$ was set to denote statistical significance.

\section{Results and Discussion}

Since wheat bran fermented by white rot fungi could hold the promise of antioxidant activity as aforementioned, its antioxidant capacity would be evaluated as free radical scavenging capacity. Anti-oxidation assays assessment methods can generally be classified into hydrogen atom transfer and electron transfer methods based on the response mechanisms. The antioxidant activity evaluation done by this study was primarily based on the electron transfer methods; the electron transfer assessment categories mainly encompassed the trolox equivalent antioxidant capacity, DPPHscavengingability, superoxide freeradicalscavenging capacity, as well as reducing power. Awika et al. (2003) compared the methods used by DPPH scavenging capacity, trolox equivalent antioxidant capacity, and total phenolic contents with the antioxidant activities of Sorghum bicolor and its extracts. The results showed that the antioxidants contained in S. bicolor exhibited a level of uniformity trend among the evaluated methods $\left(\mathrm{R}^{2}>0.96\right)$, indicating that those methods could all elaborate the antioxidant activities of the supply and tested materials. Since the DPPH is a relatively stable artificial free radical, but not superoxide anion radical, it is often used to evaluate the free radical scavenging ability for antioxidant capacity by measuring the absorbance value, in order to determine the reduced quantity, which is produced by the reduction of the DPPH by the hydrogen ions supplied by the antioxidant. In this study, the fermented wheat bran scavenging action of DPPH free radical was 1.5-fold (Figure 1) that of the wheat bran, suggesting the fermented feed displays potential antioxidant capacity. Trolox equivalent antioxidant capacity of $1 \mathrm{mg} / \mathrm{mL}$ fermented wheat bran extracts was increased from 100 to $150 \mathrm{mM}$ trolox antioxidant capacity after $12 \mathrm{~d}$ of fermentation (Figure 2). The TEAC method is dependent on the reactions between the antioxidants and oxidants. Hence, because the analysis was centered on the final reaction products, it was less influenced by the reaction rate as compared with the ferric ion reducing antioxidant method (FRAP) (Huang et al., 2005).

Compared with $0.1 \mathrm{mg} / \mathrm{mL}$ EDTA, $1.0 \mathrm{mg} / \mathrm{mL}$ fermented wheat bran extracts exerted an equivalent $50.0 \%$ chelating effect of $12 \mathrm{~d}$ fermented material (Figure 3 ). However, after $12 \mathrm{~d}$, the chelating ability only increased slightly when the wheat bran was demonstrated at $21 \mathrm{~d}$ fermentation. Free radicals were produced by the metal ions through the redox cycle and promoted acceleration of

Table 1 - Characteristics and performance data of the primers used for q-PCR analysis

\begin{tabular}{|c|c|c|c|}
\hline Gene & $\begin{array}{l}\text { Forward primer (from 5' to 3') } \\
\text { Reverse primer (from 5' to 3') }\end{array}$ & PCR product size (bp) & NCBI GenBank \\
\hline$\beta$-actin & $\begin{array}{l}\text { CTGGCACCTAGCACAATGAA } \\
\text { ACATCTGCTGGAAGGTGGAC }\end{array}$ & 109 & X00182.1 \\
\hline $\mathrm{HO}-1$ & $\begin{array}{l}\text { AGCTTCGCACAAGGAGTGTT } \\
\text { GGAGAGGTGGTCAGCATGTC }\end{array}$ & 106 & X56201.1 \\
\hline NOX1 & $\begin{array}{l}\text { CAATGCAGCACTCCACTTTG } \\
\text { GACAAGATCTCCGCAAGACC }\end{array}$ & 185 & NM_001101830.1 \\
\hline Nrf2 (NFE2L2) & $\begin{array}{l}\text { GGAAGAAGGTGCTTTTCGGAGC } \\
\text { GGGCAAGGCAGATCTCTTCCAA }\end{array}$ & 171 & NM_205117.1 \\
\hline ROMO1 & $\begin{array}{l}\text { AGCCCAGCTGCTTCGACAGAGT } \\
\text { CGTCCTCTCATGCCGATCCTGA }\end{array}$ & 115 & NM_001198821.1 \\
\hline GCLC & $\begin{array}{l}\text { CAGCACCCAGACTACAAGCA } \\
\text { CTACCCCCAACAGTTCTGGA }\end{array}$ & 118 & XM_419910.3 \\
\hline
\end{tabular}

HO-1 - heme oxygenase -1; NOX1 - NADPH oxygenase 1; GST - glutathione S-transferase; Nrf2 - nuclear factor (erythroid-derived 2)-like 2; ROMO1 - reactive oxygen species modulator protein 1; GCLC - glutamate-cysteine ligase, catalytic subunit. 
the lipid oxidation reaction. Therefore, this method only offered insights into its inhibitory effects on free radical formation (Gordon, 1990). Ebrahimzadeh et al. (2008) pointed out that ferrous ion was most frequently considered as the oxidation catalyst among various metals, causing the lipid oxidation acceleration; thus, it is usually used as an antioxidant assessment index.

Reactive oxygen species are a by-product of the constant normal oxygen metabolism of aerobic biological organisms, including $\mathrm{O}^{2-}, \mathrm{OH}^{-}$, and non-free radical $\mathrm{H}_{2} \mathrm{O}_{2}$. Reactive oxygen species induce pressure; they can be divided into endogenous and exogenous reactive oxygen species. The former refers to the mitochondrial respiratory chain and in vivo enzyme reactions, such as $\mathrm{NAD}(\mathrm{P}) \mathrm{H}$ oxidase, xanthine oxidase, lipoxygenase, and cyclooxygenases; the latter includes UV, radiation, divergent oxidizing chemicals, etc. The oxidation pressure is induced by increased reactive oxygen species, such as unbalanced reactive oxygen species affecting antioxidant system functionality, and impairs cell antioxidant capacity.
To limit potential toxic results of reactive oxygen species, the cell develops an antioxidant system, which provides two types of small molecule antioxidant agents: (a) a direct antioxidant agent that has redox activity, a short half-life, and an antioxidant function that should be regenerated or supplemented during the process; as well as (b) an indirect antioxidant agent that does not necessarily have redox activity (i.e., the cell achieves its effect by increasing the expression of antioxidant capacity genes (Jung and Kwak, 2010). If the antioxidant substance could react with the redox transcription factors, it may increase the expression of antioxidant enzymes to effectively lower cell oxidation and amplify the antioxidant reaction. The indirect antioxidant substance-activated Nrf2/Keap1/Antioxidant response element (ARE) is used to achieve antioxidant and detoxifying effects by the catalytic of transcription-induced cell protection protein (also referred to as the second phase enzyme). The Nrf2 is a transcription factor that is very sensitive to the redox reaction; it plays the role of inducing the antioxidant effect to respond to the defense mechanism
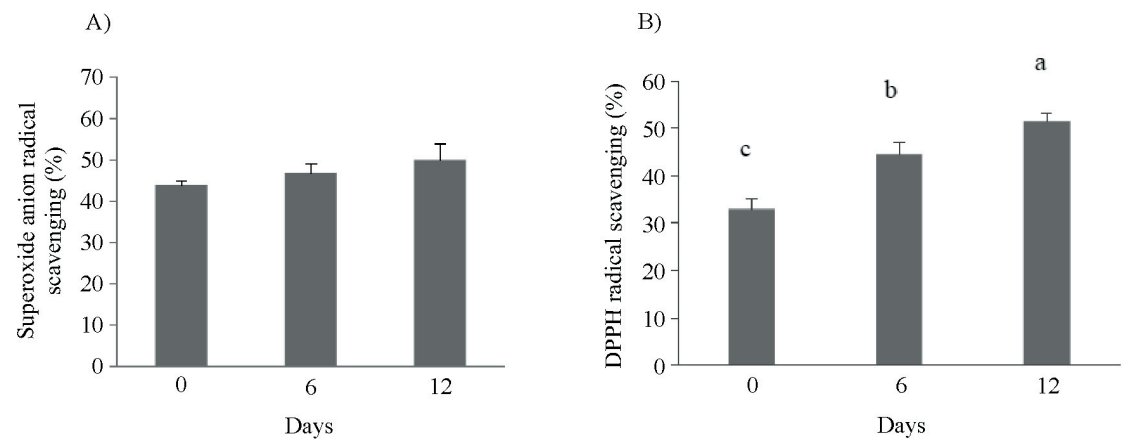

Each value represents mean \pm standard deviation $(\mathrm{n}=5)$.

DPPH - 1, 1-diphenyl-2-picrylhydrazyl.

Figure 1 - Superoxide anion (A) and DPPH (B) free radicals scavenging activity of wheat bran fermented by white rot fungi at 0 , 6 , and 12 days.

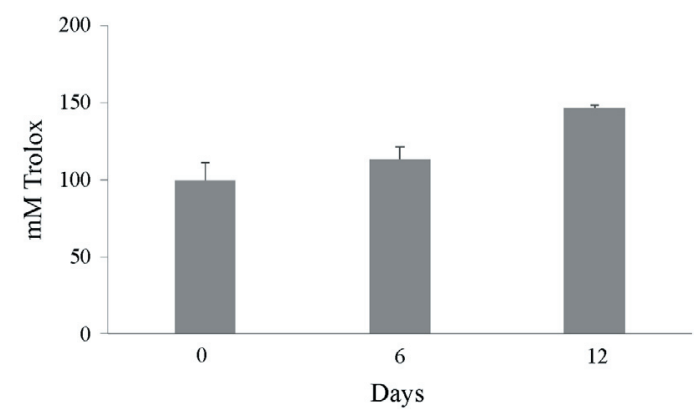

Figure 2 - Trolox equivalent antioxidant capacity of fermented wheat bran extracts.

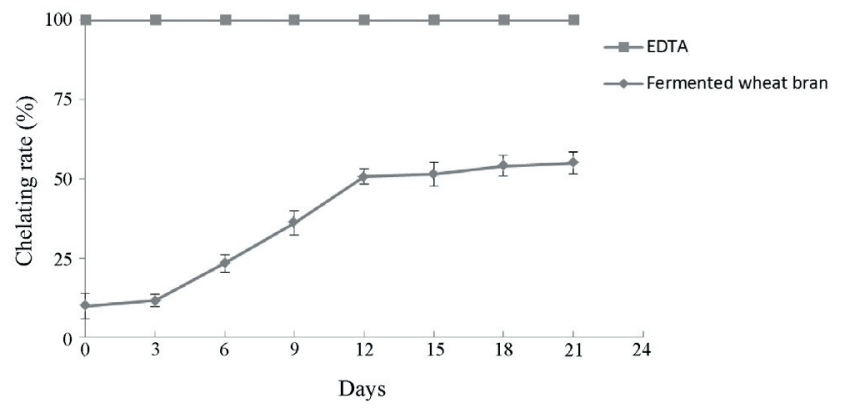

Each value represents mean \pm standard deviation $(\mathrm{n}=5)$.

Figure 3 - Fe++ chelating capacity of fermented wheat bran extracts. 
of the oxidative stimulus. Moreover, Nrf2 is present in the cytoplasm and is combined with chaperone protein molecule Keap1. When the combination of Nrf2 and Keap1 is not active, it could facilitate the hydrolysis of $\mathrm{Nrf} 2$ protein (ubiquitination), and it will be degraded (Cardozo et al., 2013). Under normal conditions, Keap1 and Nrf2 are separated by themselves. Nrf2 is sent into the nucleus for the antioxidant reaction. However, unfavorable external conditions lead to the phosphorylation of Keap1 and Nrf2. The phosphorylated Nrf2 is active and transferred into the nucleus to form the dimer form with Maf protein. Then, TIt reacts with ARE to regulate the expression of downstream antioxidant genes such as GST, GCLC, and HO-1 to achieve antioxidant and detoxifying effects (Sahin et al., 2013).

The NADPH oxidase NOX family has the function of generating reactive oxygen species. Some NOX enzymes can express relatively high tissue characteristics and generate the hormone or tissue components required to generate reactive oxygen species which cause cardiovascular disease and chronic liver/hepatitis cancer (Brandes et al., 2014; Choi et al., 2014). Most endogenous reactive oxygen species are generated in the mitochondrial respiratory chain; Romol is present in the mitochondrion and its protein increases by oxidation stimulation, thus causing the occurrence of diseases (Na et al., 2008; Chung et al., 2012). In this study, white rot fungi fermented wheat bran improved the performance of ROMO1 and NOX1 while reducing the expression, which indicates potential antioxidant effects and reduced disease occurrence (Figure 2). Glutamate-cysteine ligase (GCL) is an important synthetic enzyme of the endogenous antioxidant agent, which catalyzes the synthesis of glutamate-cysteine ligase catalytic subunit (GCLC) to start ARE. Bea et al. (2009) conducted mouse macrophage experiments and found that Nrf2 can affect specific ARE to induce increased GCLC mRNA expression. It is presumed that it can help prevent the damage caused by macrophages stimulated by oxidation. Glutamate-cysteine ligase catalytic subunit is the target gene of $\mathrm{Nrf2}$, the regulator of Nrf2 glutathione, and is an important antioxidant agent in humans (Cortese et al., 2008). Glutathione S-transferase (GST) catalyzed by glutathione can eliminate harmful and toxic compounds of the body, whereas GST can activate Nrf2 induction for detoxification (Nannelli et al., 2009). Heme oxygenase-1 (HMOX1, HO-1) responds mainly to toxicity caused by an oxidation stimulus, and can induce Nrf2 to activate the secondary detoxifying enzyme reaction (Itoh et al., 2004). Previous studies have shown that natural plant polyphenolic compounds (such as quercetin and curcumin) can activate Nrf2 to express antioxidant effects (Arredondo et al., 2010; Tanigawa et al., 2007). In addition, EGCG is a polyphenol with strong antioxidant capacity; it can improve Nrf2 and HO-1 mRNA performance expression while lowering the degree of oxidative stimulation in kidney tissues (Thangapandiyan and Miltonprabu, 2014). According to the findings of this experiment, when chicken PBMC are fermented and stimulated by antioxidant power, they can lower NOX1, ROMO1, and mRNA expression while increasing Nrf2, HO-1, GCLC, and GST expression (Figure 4). This finding suggests that fermented wheat bran has antioxidant capacity for chicken PBMC.
(A)

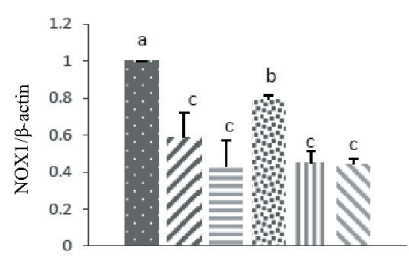

Treatments

(C)

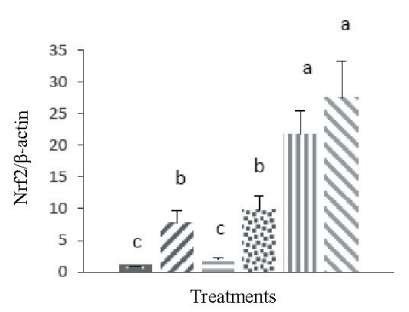

(E)

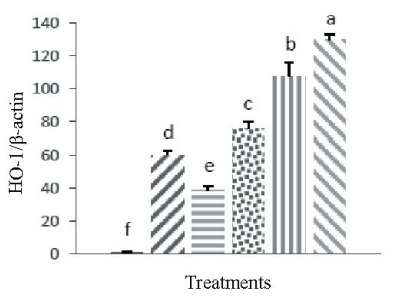

(B)

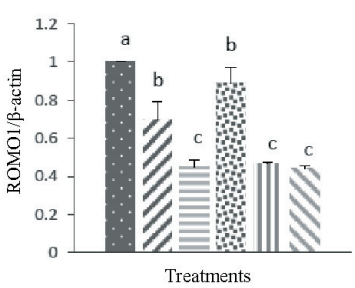

(D)

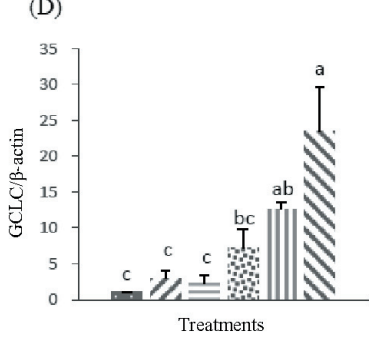

(F)

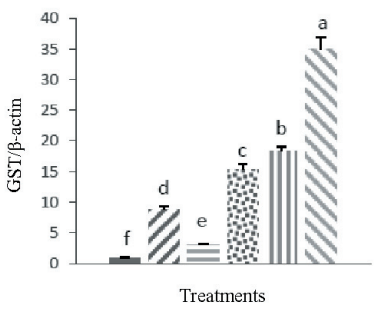

Each value represents mean \pm standard deviation $(n=5)$

Figure 4 - mRNA expression levels of antioxidant-regulated genes by PBS, vitamin C, Pleurotus eryngii hypha, wheat bran, and fermented wheat bran at 0 and 12 days in chicken peripheral blood mononuclear cells. 


\section{Conclusions}

The solid-state wheat bran fermented by white rot fungi can increase the scavenging capacity of 1, 1-diphenyl-2picrylhydrazyl, trolox equivalent antioxidant capacity, and chelating capacity of ferrous iron; in addition, in vitro, it can regulate the expression of antioxidant molecular targets in chicken peripheral blood mononuclear cells.

\section{Acknowledgments}

The authors thank the Ministry of Science and Technology of Taiwan (MOST 103-2628-B-005-001-MY3) for financially supporting this study.

\section{References}

Arredondo, F.; Echeverry, C.; Abin-Carriquiry, J. A.; Blasina, F.; Antunez, K.; Jones, D. P.; Go, Y. M.; Liang, Y. L. and Dajas, F. 2010. After cellular internalization, quercetin causes Nrf2 nuclear translocation, increases glutathione levels, and prevents neuronal death against an oxidative insult. Free Radical Biology and Medicine 49:738-747.

Awika, J. M.; Rooney, L. W.; Wu, X.; Prior, R. L. and CisnerosZevallos, L. 2003. Screening methods to measure antioxidant activity of sorghum (Sorghum bicolor) and sorghum products. Journal of Agricultural and Food Chemistry 51:6657-6662.

Bea, F.; Hudson, F. N.; Neff-Laford, H.; White, C. C.; Kavanagh, T. J.; Kreuzer, J.; Preusch, M. R.; Blessing, E.; Katus, H. A. and Rosenfeld, M. E. 2009. Homocysteine stimulates antioxidant response element-mediated expression of glutamate-cysteine ligase in mouse macrophages. Atherosclerosis 203:105-111.

Blois, M. S. 1958. Antioxidant determinations by the use of a stable free radical. Nature 181:1199-1200.

Brandes, R. P.; Weissmann, N. and Schroder, K. 2014. Redoxmediated signal transduction by cardiovascular Nox NADPH oxidases. Journal of Molecular and Cellular Cardiology 73:70-79.

Cardozo, L. F.; Pedruzzi, L. M.; Stenvinkel, P.; Stockler-Pinto, M. B.; Daleprane, J. B.; Leite, M. J. R. and Mafra, D. 2013. Nutritional strategies to modulate inflammation and oxidative stress pathways via activation of the master antioxidant switch Nrf2. Biochimie 95:1525-1533.

Choi, J.; Corder, N. L.; Koduru, B. and Wang, Y. 2014. Oxidative stress and hepatic Nox proteins in chronic hepatitis $\mathrm{C}$ and hepatocellular carcinoma. Free Radical Biology and Medicine 72:267-84.

Chung, J. S.; Park, S.; Park, S. H.; Park, E. R.; Cha, P. H.; Kim, B. Y.; Chung, Y. M.; Woo, S. R.; Han, C. J.; Kim, S. B.; Suh, K. S.; Jang, J. J.; Lee, K.; Choi, D. W.; Lee, S.; Lee, G. Y.; Hahm, K. B.; Shin, J. A.; Kim, B. S.; Noh, K. H.; Kim, T. W.; Lee, K. H. and Yoo, Y. D. 2012. Overexpression of Romol promotes production of reactive oxygen species and invasiveness of hepatic tumor cells. Gastroenterology 143:1084-1094.

Cortese, M. M.; Suschek, C. V.; Wetzel, W.; Kroncke, K. D. and Kolb-Bachofen, V. 2008. Zinc protects endothelial cells from hydrogen peroxide via Nrf2 dependent stimulation of glutathione biosynthesis. Free Radical Biology and Medicine 44:2002-2012.

Dale, N. 1996. The metabolizable energy of wheat by-products. The Journal of Applied Poultry Research 5:105-108.

Dinis, T. C. P.; Madeira, V. M. C. and Almeida, L. M. 1994. Action of phenolic derivates (acetoaminophen, salycilate, and 5-aminosalycilate) as inhibitors of membrane lipid peroxidation and as peroxyl radical scavengers. Archives of Biochemistry and Biophysics 315:161-169.

Ebrahimzadeh, M. A.; Pourmorad, F. and Bekhradnia, A. R. 2008. Iron chelating activity, phenol and flavonoid content of some medicinal plants from Iran. African Journal of Biotechnology 7:3188-3192.

Godron, M. H. 1990. The mechanism of antioxidant action in vitro. p.1-18. In: Food antioxidants. Hudson, B. J. F., ed. Elsevier Applied Science, London and New York.

Gyamfi, M. A.; Yonamine, M. and Aniya, Y. 1999. Free-radical scavenging action of medicinal herbs from Ghana: Thonningia sanguinea on experimentally-induce liver injuries. General Pharmacology 32:661-667.

Huang, D.; Ou, B. and Prior, R. L. 2005. The chemistry behind antioxidant capacity assays. Journal of Agricultural and Food Chemistry 53:1841-1856.

Itoh, K.; Tong, K. I. and Yamamoto, M. 2004. Molecular mechanism activating Nrf2 Keap1 pathway in regulation of adaptive response to elecrophiles. Free Radical Biology and Medicine 36:1208-1213.

Jung, K. A. and Kwak, M. K. 2010. The Nrf2 System as a potential target for the development of indirect antioxidants. Molecules 15:7266-7291.

Kaiser, M. G.; Cheeseman, J. H.; Kaiser, P. and Lamont, S. J. 2006. Cytokine expression in chicken peripheral blood mononuclear cells after in vitro exposure to Salmonella enterica serovar enteritidis. Poultry Science 85:1907-1911.

Lee, T. T.; Ciou, J. Y.; Chiang, C. J.; Chao, Y. P. and Yu, B. 2012. Effect of Pleurotus eryngii stalk residue on the oxidative status and meat quality of broiler chickens. Journal of Agricultural and Food Chemistry 60:11157-11163.

Lee, T. T.; Ciou, J. Y.; Chen, C. N. and Yu, B. 2014. The effect of Pleurotus eryngii stalk residue dietary supplementation on layer performance, egg traits and oxidative status. Annals of Animal Science 15:447-461.

Lin, J. T.; Liu, C. W.; Chen, Y. C.; Hu, C. C.; Juang, L. D.; Shiesh, C. C.; Yang, D. J. 2014a. Chemical composition, antioxidant and anti-inflammatory properties for ethanolic extracts from Pleurotus eryngii fruiting bodies harvested at different time. LWT - Food Science and Technology 5:374-382.

Lin, C. C.; Lin, L. J.; Wang, S. D.; Chiang, C. J.; Chao, Y. P.; Lin, J. and Kao, S. T. 2014b. The effect of serine protease inhibitors on airway inflammation in a chronic allergen-induced asthma mouse model. Mediators of Inflammation, http://dx.doi.org/10.1155/2014/ 879326.

Na, A. R.; Chung, Y. M.; Lee, S. B.; Park, S. H.; Lee, M. S. and Yoo, Y. D. 2008. A critical role for Romol-derived ROS in cell proliferation. Biochemical and Biophysical Research Communications 369:672-678.

Nannelli, A.; Rossignolo, F.; Tolando, R.; Rossato, P.; Longo, V. and Gervasi, P. G. 2009. Effect of beta-naphthoflavone on AhR regulated genes $(\mathrm{CYP} 1 \mathrm{~A} 1,1 \mathrm{~A} 2,1 \mathrm{~B} 1,2 \mathrm{~S} 1, \mathrm{Nrf} 2$, and GST) and antioxidantenzymes in various brain regions of pig. Toxicology 265:69-79.

Nishikimi, M.; Appaji, N. and Yagi, K. 1972. The occurrence of superoxide anion in the reaction of reduced phenazine methosulfate and molecular oxygen. Biochemical and Biophysical Research Communications 46:849-854.

Prückler, M.; Siebenhandl-Ehn, S.; Apprich, S.; Höltinger, S.; Haas, C.; Schmid, E. and Kneifel, W. 2014. Wheat bran-based biorefinery 1: Composition of wheat bran and strategies of functionalization. LWT - Food Science and Technology 56:211-221.

Sahin, K.; Akdemir, F.; Orhan, C.; Tuzcu, M.; Hayirli, A. and Sahin, N. 2010. Effects of dietary resveratrol supplementation on egg production and antioxidant status. Poultry Science 89:1190-1198. 
Sahin, K.; Orhan, C.; Smith, M. O. and Sahin, N. 2013. Molecular targets of dietary phytochemicals for the alleviation of heat stress in poultry. Poultry Science 69:113-124.

Sahin, N.; Orhan, C.; Tuzcu, M.; Sahin, K. and Kucuk, O. 2008. The effects of tomato powder supplementation on performance and lipid peroxidation in quail. Poultry Science 87:276-283.

Tanigawa, S.; Fujii, M. and Hou, D. X. 2007. Action of Nrf2 and Keap1 in ARE-mediated NQO1 expression by quercetin. Free Radical Biology and Medicine 42:1690-1703.

Thangapandiyan, S. and Miltonprabu, S. 2014. Epigallocatechin gallate supplementation protects against renal injury induced by fluoride intoxication in rats: Role of Nrf2/HO-1 signaling. Toxicology Reports 1:12-30.
Tuzcu, M.; Sahin, N.; Karatepe, M.; Cikim, G.; Kilinc, U. and Sahin, K. 2008. Epigallocatechin-3-gallate supplementation can improve antioxidant status in stressed quail. British Poultry Science 49:643-648

Yang, F. C.; Yang, Y. H. and Lu, H. C. 2013. Enhanced antioxidant and antitumor activities of Antrodia cinnamomea cultured with cereal substrates in solid state fermentation. Biochemical Engineering Journal 78:108-113.

Yu, B.; Liu, J. R.; Hsiao, F. S. and Chiou, P. W. S. 2008. Evaluation of transformed Lactobacillus reuteri Pg4 strain expressing heterologous $\beta$-glucanase as a probiotic in poultry diet on barley. Animal Feed Science and Technology 141:82-91. 